\title{
Synthesis of 3,3-Difluoro-2-pyrrolidone Derivatives
}

\author{
Sung-Kwan Kim. ${ }^{\dagger}$ Zhi-Feng Xie, ${ }^{\ddagger}$ Chang-Soo Jun, ${ }^{\dagger}$ Tae-Ho Kwon, ${ }^{\S}$ Soung-Ryual Ryu. ${ }^{\sharp}$ and Kyu-Yun Chai ${ }^{+\cdot \pi . *}$ \\ "Deparment of Bionanochemistry, Wonkwang Universiry, Iksan, Jeonbuk 570-749, Korea. "E-mail: geuyoon@iwonkwang.ac.k. \\ ${ }^{*}$ College of Pharmacy, Yanbin University, Yanii, Jilin 133000, P.R. China \\ \$College of Life Science and Natural Resources, Wonkwang University, Iksan, Jeonbuk 570-136, Korea \\ "Department of Chemical Engineering, Dae Bul Universiry, Chonnam 526-702, Korea \\ "Regional Innovation Center for Next Generation Industrial Radiation Technology, Jeonbuk, Iksan 570-749, Korea \\ Received Augtist 10, 2007
}

\begin{abstract}
Introduction of a ditluoromethylene group into organic compounds has been observed to impart them with positive properties, as viewed by a wide range of industries. Here, synthesis of 3,3-difluoro-2-pyrrolidone derivatives (7) was accomplished by the reaction of ethyl 2,2-difluoro-4-iodo-4-(trimethylsilyl) butanolate (4) with primary amines followed by desilylation. The key internediate (4) was prepared from the addition reaction of trimethylvinylsilane (3) to ethyl difluoroiodoacetate (2) in the presence of $\mathrm{Cu}(0)$. Ethyl difluoroiodoacetate (2) was prepared starting from ethyl bromodifluoroacetate (1) via Reformatsky-type reaction.
\end{abstract}

Key Words : 3,3-Difluoro-2-pyrrolidone, $\alpha, \alpha$-Difluoro-;-lactam, Difluoromethylene group

\section{Introduction}

Organic compounds containing a difluoromethylene group often display significant physiological properties, such as antibiotic, ${ }^{2}$ and an inhibitory effect on various enzymes, ${ }^{1}$ the human immunodeficiency virus, or $\mathrm{HIV}^{3}$ cancers and hypertension. ${ }^{4}$ These desirable properties stem from difluoromethylene's strong carbon-fluorine bond and its lipophilicity.

In general, we incorporated a difluoromethylene group into organic compounds, using the following reactions: (a) the addition of perfluoroalkyl iodide to alkenes, performed in the presence of various catalysts, such as titanium, ${ }^{5}$ benzoylperoxide, ${ }^{6}$ sodium dithionite ${ }^{7}$ and organophosphines ${ }^{8}$ (b) the Reformatsky reaction, which was used to prepare $\alpha, \alpha$-difluoro- $\beta$-hydroxy carbonyl compounds from the reaction of aldehydes with $\alpha$-chloro- $\alpha, \alpha$-difluoromethyl ketones 9 or $\alpha$-bromo- $\alpha, \alpha$-difluoro acetates ${ }^{10}$ (c) the addition of ethyl bromodifluoroacetate to alkenes, carried out using nickel chloride or copper powder as a catalyst. ${ }^{11}$ (d) the transformation of carbonyl group to difluoromethylene group, utilizing (diethylamino)sulfur trifluoride, or DAST. ${ }^{12}$ Researchers recently reported that difluoromethylene ketone could be introduced into the electron-deficient olefins, using UV irradiation ${ }^{13}$ and that palladium catalyst has been used for difluoromethylene ketone addition to electron-rich olefins. $^{1+}$

Derivatives of pyrrolidones comprising peripheral difluoromethylene are also regarded as key building blocks for biologically important compounds. Naturally occurring pyrrolidones have been found to be anti-microbial, anti-tumor, and anti-histamine. $^{15}$

Brahms and Dailey reported that although difluoromethylene resembles methylene in appearance, compounds possessing difluoromethylene show different properties from those possessing methylene. ${ }^{16}$ And they observed that while it is well known that fluorine is the most electronegative element, the idea that fluorine can donate electron density to a $\pi$ system, using one of its lone pairs, is not as well appreciated. For instance, fluorine can act as a slightly activating ortho-para director in electrophilic aromatic substitution, and the substitution reaction on nitrogen in 3,3difluoro-2-pyrrolidone, which is in a meta-position, would not be preferred in mild reaction conditions. ${ }^{16}$ Recently, Eguchi et al. performed cyclization of difluoro-GABA via Staudinger/intramolecular-aza-Wittig approach of its azidoester with trialkylphosphine. ${ }^{15}$ The reaction of azoester with $\mathrm{PPh}_{3}$ in toluene at room temperature gave $\alpha, \alpha$-difluorobutanlactam in $c a .36 \%$ yield. There are, in addition, only a few examples of difluoromethylene bearing $\gamma$-lactams reported. ${ }^{17.18}$

It is noted that a direct substitution reaction on the 3,3difluoro-2-pyrrolidone should be avoided in developing a high-yield synthetic route. Therefore, we adapted a cyclization method that could incorporate various substituents of biological importance to the nitrogen atom of pyrrolidone during ring formation. That is, ethyl 2,2-difluoro-4-iodo-4(trimethylsilyl)butanolate (4) and primary amines are allowed to react for the preparation of $N$-substituted difluoropyrrolidones ${ }^{19}$ (Figure 1). In the present report, we detail the synthesis of a series of noble $N$-alkyl 3,3-difluoro-2pyrrolidon derivatives.

\section{Results and Discussion}

Eguchi et al. synthesized 3,3-difluoro-2-pyrrolidone from methyl 4-azido-2,2-difluorobutanoate via ring formation by $\mathrm{PPh}_{3}$ or $\mathrm{PBu}_{3 .}{ }^{17}$ When the reaction was proceeded in toluene with $\mathrm{PPh}_{3}$, an intemediate, 4,4-difluoro-3,4-dihydro-5methoxy- $2 H$ pyrrole, was formed first. But, it was prone to 


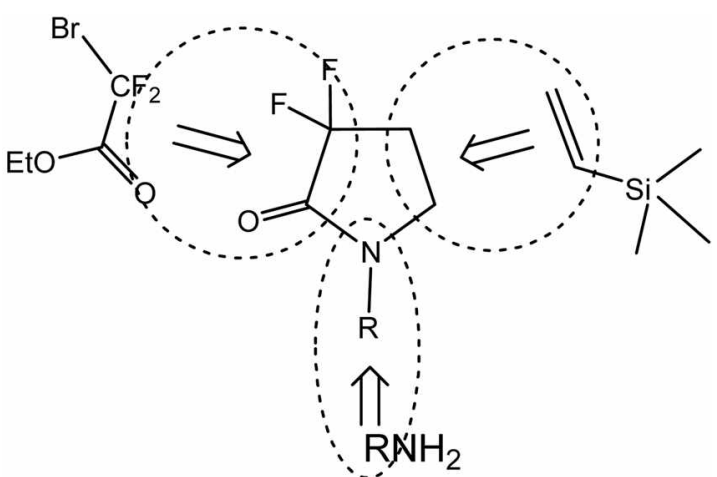

Figure 1. Retrosynthesis of I-Alkyl- $\alpha, \alpha$-difiuoro-2-pyrrolidone.

be hydrolyzed to yield 3,3-difluoro-2-pyrrolidone. Use of more nucleophilic $\mathrm{PBu}_{3}$ in dried $\mathrm{THF}$ gave only 3,3difluoro-1-methyl-2-pyrrolidone without an intermediate. Based on this finding, it is expected that further variation of the -OR group in alkyl 4-azido-2,2-difluorobutanoate could yield $N$-substituted 3,3-difluoro-2-pyrrolidone. However, use of azid derivatization and intrinsic low yields are significant drawbacks in practice applications.

In our retrosynthetic analysis of 3,3-difluoro-2-pyrrolidone, ethyl iododifluoroacetate, trimethyl(vinyl)silane and primary amines were chosen as potential starting materials. (Figure 1). Reaction of ethyl bromodifluoroacetate, $\mathrm{Zn}, \mathrm{I}$, and $\mathrm{HgCl}_{2}$ in triglyme solution gave ethyl iododifluoroacetate in $64 \%$ yield, ${ }^{20}$ but we encountered a further problem removing the solvent. Under acetonitrile solvent, however, the same reaction required only $\mathrm{Zn}, \mathrm{I}_{2}$ at $0^{\circ} \mathrm{C}$ to give ethy] jododifluoroacetate in $90 \%$ yie]d.

Addition reaction of ethyl iododifluoroacetate (2) to viny] trimethylsilane $(3)$ under the $\mathrm{Cu}(0)$ catalyst resulted in the formation of ethyl 2,2-difluoro-4-iodo-4-(trimethylsilyl)butanolate (4) in $91 \%$ yield. The reaction involves a radical mechanism in which a single electron transfer occurs, as reported by Yang $e t a l^{17}$ Then, (4) reacted with various primary amines (5) to yield $N$-alkyl-3,3-difluoro-5-trimethylsilyl-2-pyrrolidones (6) (Scheme 1). These results are
Table 1. Preparation of $N$-alkyl-3,3-difluoro-5-truncthylsılyl-2pyrrolidones (6) and $N$-alkyl-3,3-difluoro-2-pyrrolidones (7)

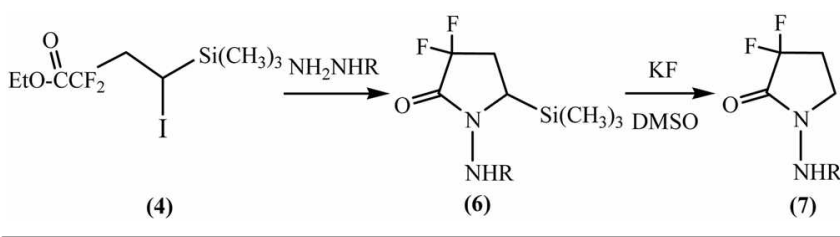

\begin{tabular}{ccccccc}
\hline Entry Substrate & Reactants $\left(\mathrm{R}-\mathrm{NH}_{2}\right)$ & (6) & $\begin{array}{c}\text { Yield of } \\
(6)^{*}\end{array}$ & $(7)$ & $\begin{array}{c}\text { Yield of } \\
(7)^{*}\end{array}$ \\
\hline 1 & $\mathbf{4}$ & $\mathrm{H}$ & $6 \mathrm{a}$ & 72 & - & - \\
2 & $\mathbf{4}$ & $n-\mathrm{C}_{4} \mathrm{H}_{9}$ & $6 \mathrm{~b}$ & 70 & $7 \mathrm{~b}$ & 74 \\
3 & $\mathbf{4}$ & $\mathrm{Cyylohexyl}_{2}$ & $6 \mathrm{c}$ & 81 & $7 \mathrm{c}$ & 75 \\
4 & $\mathbf{4}$ & $\mathrm{CH}_{2} \mathrm{C}_{6} \mathrm{H}_{3}$ & $6 \mathrm{~d}$ & 67 & $7 \mathrm{~d}$ & 70 \\
5 & $\mathbf{4}$ & $\mathrm{CH}_{2} \mathrm{CH}_{2} \mathrm{C}_{6} \mathrm{H}_{5}$ & $6 \mathrm{e}$ & 75 & $7 \mathrm{e}$ & 80 \\
6 & 4 & $\mathrm{CH}_{2} \mathrm{C}_{6} \mathrm{H}_{4}(m-\mathrm{F})(p-\mathrm{F})$ & $6 \mathrm{f}$ & 71 & $7 \mathrm{f}$ & 79 \\
7 & $\mathbf{4}$ & $\mathrm{CH}_{2} \mathrm{C}_{6} \mathrm{H}_{5}(o-\mathrm{F})$ & $6 \mathrm{~g}$ & 72 & $7 \mathrm{~g}$ & 75 \\
8 & 4 & $\mathrm{CH}_{2} \mathrm{C}_{6} \mathrm{H}_{5}(p-\mathrm{F})$ & $6 \mathrm{~h}$ & 77 & $7 \mathrm{~h}$ & 82 \\
\hline
\end{tabular}

summarized in Table 1. As can be seen, the smaller alkyl primary amines the better the yields. This is a result of a steric effect between the bulky trimethylsilyl group and the alkyl group of amine. Removal of the trimethylsilyl group occurred by the addition $\mathrm{KF}$. The reaction of (6) with $\mathrm{KF}$ at $100{ }^{\circ} \mathrm{C}$ resulted in $\mathrm{N}$-alkyl-3,3-difluoro-2-pyrrolidones in good yields (Table 1 ).

All the products were identified using ${ }^{1} \mathrm{H} N \mathrm{NR},{ }^{13} \mathrm{C} N M R$, ${ }^{19} \mathrm{~F}$ NMR and MS spectra. ${ }^{19} \mathrm{~F}$ NMR spectra of all the adducts showed typical $\mathrm{AB}$ splitting pattens, because the two fluorines are not equivalent because of the presence of the $\gamma$-positioned chiral center. For instance, ${ }^{2} \mathrm{~J}_{\mathrm{TT}}$ coupling constant of (6) observed in ${ }^{19} \mathrm{~F}-\mathrm{NMR}$ showed a typically high value of $262 \mathrm{~Hz}$, which would reflect diasterotopic fluorine atoms on the ring system. When trimethyl groups were removed, therefore, the coupling constants between fluorine atoms in (7) appeared as nullified, giving a singlet ${ }^{19} \mathrm{~F}-\mathrm{NMR}$ signal. This would imply that fluorine atoms in 3,3-difluoro-2-pyrrolidone (7) become enantiotopic atoms as

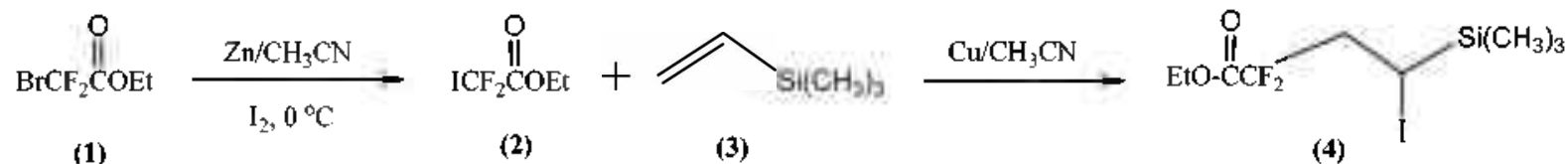

(1)

(3)<smiles>[R]N1C(=O)C(F)(F)CC1[SiH2][14CH3]</smiles><smiles>CO[C@H](C)O[Na]</smiles><smiles>CC(F)=C(C)C(F)F</smiles><smiles>[R]N1CCC(F)(F)C1=O</smiles>

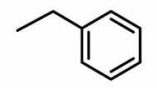

(d)

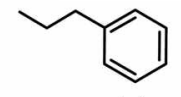

(e) (f)<smiles>CCc1ccc(F)c(F)c1</smiles>

R: $\quad-H$

(a) (b)

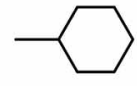

(c)<smiles>CCc1ccccc1F</smiles>

(g)<smiles>CCc1ccc(F)cc1</smiles>

(h)

Scheme 1 
the chiral center (trimethylsilyl group) was removed.

\section{Conclusions}

Ethyl 2,2-difluoro-4-iodo-4-(trimethylsilyl) butanolate (4) was reacted with primary amines (5) to yield a series of $N$ alkyl-3,3-difluoro-5-trimethylsilyl-2-pyrrolidones (6), which was reacted further with $\mathrm{KF}$ at $100^{\circ} \mathrm{C}$ to result in the formation of $\mathrm{N}$-alkyl-3,3-difluoro-2-pyrrolidones(7) via the removal of the trimethylsilyl group. Our synthetic platform appears suitable for preparing noble fluorinated lactam derivatives to explore their highly branched and fluorinated analogues for pharmaceutical applications.

\section{Experimental}

General. ${ }^{19} \mathrm{~F}$ NMR were recorded on a Jeol JNM-ECP $500 \mathrm{MHz})$ or Bruker AC $-300(282.44 \mathrm{MHz})$. Spectra were recorded on the $\mathrm{AC}-300$ spectrometer. All samples were taken in $\mathrm{CDCl}_{3}$ solvent and all chemical shifts are reported in parts per million downfield (positive) of the standard: TMS of ${ }^{1} \mathrm{H}$ and ${ }^{13} \mathrm{C} ; \mathrm{CFCl}_{3}$ for ${ }^{19} \mathrm{~F}$ NMR. FT-IR spectra were recorded as $\mathrm{CCl}_{4}$ solutions and reported in wavenumber $\left(\mathrm{cm}^{-1}\right)$. GC-MS spectra were obtained at $70 \mathrm{eV}$ in the electron impact mode (Shimadzu GC 17A-QP5000). Infrared spectra were obtained with a Jasco FT/IR-5300 Spectrophotometer.

Preparation of Cu(0) catalyst. To a $500 \mathrm{~mL}$ Erlenmeyer flask [S2] was introduced $200 \mathrm{~mL}$ of carbon tetrachloride, $40 \mathrm{~g}$ of copper powder and $15 \mathrm{~g}$ of iodine. The solution was stirred until it was colorless and filtered with a Buchner funnel. The $\mathrm{Cu}(0)$ catalyst obtained was washed with a solution of acetone and concentrated hydrochloric acid $(500$ $\mathrm{mL}, 1: 1)$ followed by rinsing with acetone 3 to 5 times. It was then dried before being stored in a dried bottle under nitrogen.

Ethyl 2,2-difluoro-4-iodo-4-(trimethylsilyl)butanoate (4). $12.5 \mathrm{~g}$ of ethyl iododifluoro-aceate $(50.00 \mathrm{mmol}), 10.00$ $\mathrm{g}$ of trimethylvinylsilane $(100.0 \mathrm{mmol})$ and $2.5 \mathrm{~g}$ of activated copper powder $(2.5 \mathrm{mmol})$ were added to $200 \mathrm{~mL}$ of acetonitrile dried over phosphorus pentoxide. The reaction mixture was stirred $15 \mathrm{hrs}$ at $65^{\circ} \mathrm{C}$. The reaction mixture was purified by flash column chromatography (silica gel $60 \mathrm{~F}-254$, ethyl acetate: $n$-hexane $=1: 3, \mathrm{R}_{f}=0.68$ ) to afford ethyl 2,2-difluoro-4-iodo-4-(trimethylsilyl)butanoate $(15.6 \mathrm{~g}, 91 \%)$ as a colorless liquid.

${ }^{1} \mathrm{H}$ NMR $\left(\mathrm{CDCl}_{3}\right) \delta: 0.18(\mathrm{~s}, 9 \mathrm{H}), 1.38(\mathrm{t}, 7.1 \mathrm{~Hz}, 3 \mathrm{H})$, $2.60(\mathrm{~m}, 2 \mathrm{H}), 3.11(\mathrm{t}, 6.6 \mathrm{~Hz}, 1 \mathrm{H}), 4.36(\mathrm{q}, 7.1 \mathrm{~Hz}, 2 \mathrm{H}) .{ }^{13} \mathrm{C}$ NMR $\left(\mathrm{CDCl}_{3}\right) \delta:-2.42,4.41(\mathrm{t}, 4.2 \mathrm{~Hz}), 13.94,39.16(\mathrm{t}$, $24.2 \mathrm{~Hz}), 63.19,115.74(\mathrm{t}, 252.6 \mathrm{~Hz}), 163.77(\mathrm{t}, 31.9 \mathrm{~Hz})$. ${ }^{19} \mathrm{~F}$ NMR $\left(\mathrm{CDCl}_{3}\right) \delta .-108.6(\mathrm{dt}, J=73.0 \mathrm{~Hz}, 17.1 \mathrm{~Hz})$, -102.3 (ddd, $J=261 \mathrm{~Hz}, 14.6 \mathrm{~Hz}, 12.2 \mathrm{~Hz}$ ). FT-IR $\left(\mathrm{CCl}_{4}\right)$ : $1095(\mathrm{~s}), 1191$ (s), 1254 (s), 1761 (s), 1744 (s), $2960(\mathrm{~m})$ $\mathrm{cm}^{-1}$ GC-MS $\mathrm{m} / \mathrm{z}$ (relative intensity): $73(78.0), 77(53.7)$, 84 (25.7), 103 (100), 185 (5.5), $350\left(\mathrm{M}^{+} 2.3\right)$. HRMS: C9H21O2F2ISi, Calculated, 354.03237; Observed, 354.03193.

3,3-Difluoro-5-trimethylsilyl-2-pyrrolidone (6a). $1.00 \mathrm{~g}$ of ethyl 2,2-difluoro-4-iodo-4-(trimethylsilyl)butanoate $(2.80 \mathrm{mmol})$ was added to $4.50 \mathrm{~mL}$ of aqueous ammonium hydroxide solution $(28 \%, 4.70 \mathrm{mmol})$ and stirred for $24 \mathrm{hrs}$. The reaction products were extracted with diethyl ether and separated by TLC (alumina 60F, ethyl acetate:n-hexane = $\left.1: 3, \mathrm{R}_{\mathrm{r}}=0.67\right) \cdot 0.42 \mathrm{~g}$ of $6 \mathrm{a}$ was isolated as a colorless liquid. (Yield: $72 \%$ ) To prepare $6 \mathbf{b}$ to $6 \mathbf{h}$, the same procedure was applied varying only the amines, and the yields of products are noted in Table 1.

'H NMR $\left(\mathrm{CDCl}_{3}\right) \&: 0.11(\mathrm{~s}, 9 \mathrm{H}), 2.25(J=8.3,15.5,15.5$, $22.5 \mathrm{~Hz}, 1 \mathrm{~Hz}), 2.59(J=6.9,6.9,14.2,18.3 \mathrm{~Hz}, 1 \mathrm{~Hz}), 3.12$ (ddd, $J=7.4,7.4,1.8 \mathrm{~Hz}, 1 \mathrm{~Hz}), 7.79(\mathrm{~s}) .{ }^{13} \mathrm{C} \mathrm{NMR}\left(\mathrm{CDCl}_{3}\right)$ $\delta:-4.4,32.9(\mathrm{t}, 22.4 \mathrm{~Hz}), 39.1,118.3(\mathrm{t}, 259.4 \mathrm{~Hz}), 167.3(\mathrm{t}$, $30.7 \mathrm{~Hz}$ ). ${ }^{19} \mathrm{~F} \mathrm{NMR}\left(\mathrm{CDCl}_{3}\right) \delta:-108.4(\mathrm{ddd}, J=266.5,15.0$, $6.4 \mathrm{~Hz}),-106.9$ (ddd, $J=266.5,19.3,19.3 \mathrm{~Hz})$. FT-IR $\left(\mathrm{CCl}_{4}\right)$ : $1256(\mathrm{~s}), 1427(\mathrm{~m}), 2961(\mathrm{~s}) \mathrm{cm}^{-1}$. GC-MS m/z (relative intensity): $55.05(19.90), 73.00(100), 115.00(38.34), 191.95$ $(\mathrm{M}+, 1.94), 192.95\left(\mathrm{M}^{+}+1,0.38\right)$. HRMS: C7H13NOF2Si, Calculated, 193.07345; Observed, 193.07237.

1-Butyl-3,3-difluoro-5-trimethylsilyl-2-pyrrolidone (6b). 'H NMR (CDCl $) \&: 0.14(\mathrm{~s}, 9 \mathrm{H}), 0.92(\mathrm{t}, 5.4 \mathrm{~Hz}, 3 \mathrm{H}), 1.30$ $(\mathrm{m}, 2 \mathrm{H}), 1.53(\mathrm{~m}, 2 \mathrm{H}) 2.56$ (dddd, $J=8.0,10.5,14.6,17.0$ $\mathrm{Hz}, 1 \mathrm{H}$ ), 2.24 (dddd, $J=7.114 .5,16.5,17.9 \mathrm{~Hz}, 1 \mathrm{H}$ ), 2.87 (dddd, $J=2.0,4.9,6.8,8.8 \mathrm{~Hz}, 1 \mathrm{H}$ ), 3.20 (ddd, $J=2.3,7.7$ $7.7 \mathrm{~Hz}, 1 \mathrm{H}$ ), 3.90 (ddd, $J=7.9,13.6,16.4 \mathrm{~Hz}, 1 \mathrm{H}$ ). ${ }^{13} \mathrm{C}$ $\operatorname{NMR}\left(\mathrm{CDCl}_{3}\right) \delta .-3.0,13.9,20.0,28.9,32.0(\mathrm{t}, 23.0), 42.8$, $43.3,118(\mathrm{t}, 249.5), 164.19(\mathrm{t}, 30.7) .{ }^{19} \mathrm{~F} \mathrm{NMR}\left(\mathrm{CDCl}_{3}\right) \delta$ : -106.2 (ddd, $J=266.0,10.3,17.6 \mathrm{~Hz}$ ), -105.4 (ddd, $J=$ $266.0,17.6,17.6 \mathrm{~Hz})$. FT-IR $\left(\mathrm{CCl}_{4}\right): 1259(\mathrm{~s}), 1427(\mathrm{~m})$, 1722, $2966(\mathrm{~s}) \mathrm{cm}^{-1}$. GC-MS m/z (relative intensity): 57 (27.33) $73.00(100), 86(13.99), 120(0.30), 128(2.86), 250$ $\left(\mathrm{M}^{+}, 0.09\right)$. HRMS: C11H21NOF2Si, Calculated, 249.13605; Observed, 249.13442.

1-Cyclohexyl-3,3-difluoro-5-trimethylsilyl-2-pyrrolidone (6c). ${ }^{1} \mathrm{H}$ NMR $\left(\mathrm{CDCl}_{3}\right) \delta: 0.15(\mathrm{~s}, 9 \mathrm{H}), 1.14-1.24(\mathrm{~m}, 3 \mathrm{H})$, $2.04-1.60(\mathrm{~m}, 6 \mathrm{H}), 2.34-2.23(\mathrm{~m}, 2 \mathrm{H}), 2.55(\mathrm{~m}, 1 \mathrm{H}), 3.06$ $(\mathrm{m}, 1 \mathrm{H}), 3.15(\mathrm{~m}, 1 \mathrm{H}) .{ }^{13} \mathrm{C} \mathrm{NMR}\left(\mathrm{CDCl}_{3}\right) \delta:-2.61(3 \mathrm{c})$, $24.97,25.90,26.0,28.38,28.94,32.34$ (t, 23.0 Hz), 44.85, $58.15,118.24(\mathrm{t}, 249.5 \mathrm{~Hz}), 163.4(\mathrm{t}, 30.7 \mathrm{~Hz}) .{ }^{19} \mathrm{~F}$ NMR $\left(\mathrm{CDCl}_{3}\right) \delta$-104.98 (ddd, $J=263.5,12.6,18.0 \mathrm{~Hz}$ ), -102.88 (ddd, $J=263.5,12.6,18.0 \mathrm{~Hz}$ ). FT-IR $\left(\mathrm{CCl}_{4}\right): 1286(\mathrm{~s}), 1429$ (m), $1718(\mathrm{~m}), 2962(\mathrm{~s}), 3156(\mathrm{~s}) \mathrm{cm}^{-1}$. GC-MS m/z (relative intensity): 55.05 (72.13) 73.00 (100), 100.95 (81.96), 192.05 (25.17), $275.0\left(1.98, \mathrm{M}^{+}\right), 276.05$ (1.24). HRMS: C13H23NOF2Si, Calculated, 275.15170; Observed, 275.15091.

1-Benzyl-3,3-difluoro-5-trimethylsilyl-2-pyrrolidone (6d). 'H NMR $\left(\mathrm{CDCl}_{3}\right) \delta: 0.11(\mathrm{~s}, 9 \mathrm{H}), 2.31$ (ddd, $J=30.7,15.7$, $6.4 \mathrm{~Hz}, 1 \mathrm{H}), 2.53$ (ddd, $30.7,15.7,8.7 \mathrm{~Hz}, 1 \mathrm{H}), 3.09$ (dd, $J=$ 8.7, 6.4 Hz, 1H), 3.92 (d, $15.4 \mathrm{~Hz}, 1 \mathrm{H}), 5.30$ (d, $15.4 \mathrm{~Hz}$, $1 \mathrm{H}), 7.16(\mathrm{~d}, J=7.4 \mathrm{~Hz}, 1 \mathrm{H}), 7.33(\mathrm{~m}, 4 \mathrm{H}) \cdot{ }^{13} \mathrm{C}$ NMR $\left(\mathrm{CDCl}_{3}\right) \& \mathrm{t}-2.9(\mathrm{~s}), 32.0(\mathrm{t}, 23.0 \mathrm{~Hz}), 42.6,47.0,118.3(\mathrm{t}$, $249.5 \mathrm{~Hz}), 127.7,128.2,129.1,134.8,164.6(\mathrm{t}, 30.7 \mathrm{~Hz}) .{ }^{19} \mathrm{~F}$ NMR $\left(\mathrm{CDCl}_{3}\right) \delta:-105.39$ (ddd, $J=265.0,15.7,15.7 \mathrm{~Hz}$ ), -104.82 (ddd, $J=265.0,15.7,15.7 \mathrm{~Hz}$ ). FT-IR $\left(\mathrm{CCL}_{4}\right): 1258$ (s), $1431(\mathrm{~m}), 1722(\mathrm{~m}), 2957(\mathrm{~s}) \mathrm{cm}^{-1}$. GC-MS m/z (relative intensity): 73.05 (100) $91.00(88.40), 192.05$ (34.54), 282.95 $\left(\mathrm{M}^{+}, 0.79\right), 283.95\left(\mathrm{M}^{+}+1,0.13\right)$. HRMS: C14H19NOF2Si, 
Calculated, 283.12040; Observed, 283.11998.

$N$-(2-Phenylethyl)-3,3-difluoro-5-trimethylsilyl-2-pyrrolidone (6e). ' $\mathrm{H}$ NMR ( $\mathrm{CDCl}_{3}$, TMS): $\delta 0.16(\mathrm{~s}, 9 \mathrm{H}), 2.47-$ $2.75(\mathrm{~m}, 2 \mathrm{H}), 2.87(\mathrm{t}, J=6.9 \mathrm{~Hz}, 2 \mathrm{H}), 2.97(\mathrm{dd}, J=11.5,2.8$ $\mathrm{Hz}, 1 \mathrm{H}), 3.59-3.63(\mathrm{~m}, 2 \mathrm{H}), 7.21-7.32(\mathrm{~m}, 5 \mathrm{H}) .{ }^{13} \mathrm{C} \mathrm{NMR}$ $\left(\mathrm{CDCl}_{3}, \mathrm{TMS}\right): \delta-2.37(\mathrm{~s}), 4.90(\mathrm{~s}), 35.29(\mathrm{~s}), 37.79(\mathrm{t}, J=$ $23.8 \mathrm{~Hz}$ ), 40.75 (s), 117.74 (t, $J=254.4 \mathrm{~Hz}), 126.90$ (s), $128.83(\mathrm{~s}), 128.88(\mathrm{~s}), 138.09(\mathrm{~s}), 163.71(\mathrm{t}, J=38.4 \mathrm{~Hz}) .{ }^{19} \mathrm{~F}$ NMR $\left(\mathrm{CDCl}_{3}, \mathrm{CFCl}_{3}\right): \delta-104.05$ (dd, $J=253.0,23.0 \mathrm{~Hz}$, $1 \mathrm{~F}),-105.58$ (dd, $J=253.0,23.0,1 \mathrm{~F})$. FT-IR $\left(\mathrm{CCl}_{4}\right): 2955$, $1693,1680,1542,1442,1205,1188,1082 \mathrm{~cm}^{-1}$. HRMS: C15H21NOF2Si, Calculated, 297.13605; Observed, 297.13584.

N-(3,4-Difluorobenzyl)-3,3-difluoro-5-trimethylsilyl-2pyrrolidone (6f). 'H NMR ( $\left.\mathrm{CDCl}_{3}, \mathrm{TMS}\right)$ : $\delta 7.13-7.15$ (m, $3 \mathrm{H}), 4.46(\mathrm{~m}, 2 \mathrm{H}), 3.00(\mathrm{dd}, J=11.9 \mathrm{~Hz}, 2.3 \mathrm{~Hz}, 1 \mathrm{H}), 2.58-$ $2.76(\mathrm{~m}, 2 \mathrm{H}), 0.14(\mathrm{~s}, 9 \mathrm{H}) .{ }^{13} \mathrm{C} \mathrm{NMR}\left(\mathrm{CDCl}_{3}, \mathrm{TMS}\right): \delta$ $163.86(\mathrm{t}, J=28.6 \mathrm{~Hz}$ ), 150.55 (dd, $J=248.0 \mathrm{~Hz}, J=12.4$ $\mathrm{Hz}), 150.19(\mathrm{dd}, J=248.0 \mathrm{~Hz}, J=12.4 \mathrm{~Hz}), 133.74$ (t, $J=$ $3.8 \mathrm{~Hz}), 124.13(\mathrm{dd}, J=6.6 \mathrm{~Hz}, J=3.8 \mathrm{~Hz}), 118.78(\mathrm{~s})$, $117.84(\mathrm{t}, J=253.7 \mathrm{~Hz}), 117.81(\mathrm{t}, J=17.2 \mathrm{~Hz}), 117.15(\mathrm{~d}, J$ $=18.1 \mathrm{~Hz}), 42.83(\mathrm{~s}), 37.78(\mathrm{t}, J=23.8 \mathrm{~Hz}), 29.79(\mathrm{~s})$, -2.39 (s). ${ }^{19} \mathrm{~F} \mathrm{NMR}\left(\mathrm{CDCl}_{3}, \mathrm{CFCl}_{3}\right): \delta-104.12$ (dd, $J=$ $252.4,23.3 \mathrm{~Hz}, 1 \mathrm{~F}),-105.66$ (dd, $J=252.4,23.3,1 \mathrm{~F})$. IR (KBr); 2955, 1687, 1562, 1493, 1251, 1197, $1105 \mathrm{~cm}^{-1}$. GCMS (m/z, relative intensity): HRMS: C14H17NOF4Si, Calculated, 319.10156; Observed, 319.10147.

$N$-(2-Fluorobenzyl)-3,3-difluoro-5-trimethylsilyl-2-pyrrolidone $(6 \mathrm{~g}) .{ }^{1} \mathrm{H}$ NMR $\left(\mathrm{CDCl}_{3}\right.$, TMS): $\delta 0.11(\mathrm{~s}, 9 \mathrm{H}), 2.42-$ $2.51(\mathrm{~m}, 2 \mathrm{H}), 3.36(\mathrm{t}, J=6.5 \mathrm{~Hz}, 1 \mathrm{H}), 4.58(\mathrm{~s}, 2 \mathrm{H}), 7.04-$ $7.36(\mathrm{~m}, 4 \mathrm{H}) .{ }^{13} \mathrm{C}$ NMR (CDCl, TMS $): \delta-2.39(\mathrm{~s}), 29.80$ (s), 37.86 (t, $J=23.8 \mathrm{~Hz}), 43.88(\mathrm{~s}), 115.76(\mathrm{~d}, J=21.0 \mathrm{~Hz}$ ), $117.79(\mathrm{t}, J=243.2 \mathrm{~Hz}), 123.76(\mathrm{~d}, J=15.2 \mathrm{~Hz}), 124.53(\mathrm{~d}$, $J=3.8 \mathrm{~Hz}), 128.38(\mathrm{~s}), 130.61(\mathrm{~d}, J=3.8 \mathrm{~Hz}), 162.22(\mathrm{t}, J=$ $253.7 \mathrm{~Hz}), 163.77(\mathrm{t}, J=28.6 \mathrm{~Hz}) .{ }^{19} \mathrm{~F}$ NMR $\left(\mathrm{CDCl}_{3}\right.$, $\left.\mathrm{CFCl}_{3}\right): \delta-104.38$ (dd, $\left.J=253.0,34.5 \mathrm{~Hz}, 1 \mathrm{~F}\right),-105.58$ (dd, $J=253.0,34.5 \mathrm{~Hz}, 1 \mathrm{~F})$. R (KBr); 1105, 1197, 1251, $1493,1562,1687,2955 \mathrm{~cm}^{-1}$. GC-MS $(\mathrm{m} / \mathrm{z}$, relative intensity): 45 (11.70), 73 (30.91), 83 (12.04), $109(100.0), 140$ (7.69), $210(4.10), 275(4.54), 301\left(\mathrm{M}^{+}, 3.37\right)$. HRMS: C14H17NOF4Si, Calculated, 301.11098; Observed, 301.11137.

$\mathrm{N}$-(4-Fluorobenzyl)-3,3-difluoro-5-trimethylsilyl-2-pyrrolidone (6h). 'H NMR ( $\mathrm{CDCl}_{3}$, TMS): $\delta 7.02-7.29(\mathrm{~m}, 4 \mathrm{H})$, $4.43-4.53(\mathrm{~m}, 2 \mathrm{H}), 3.01(\mathrm{dd}, J=11.5 \mathrm{~Hz}, 2.3 \mathrm{~Hz}, 1 \mathrm{H}), 2.54-$ $2.79(\mathrm{~m}, 2 \mathrm{H}), 0.16(\mathrm{~s}, 9 \mathrm{H}) .{ }^{13} \mathrm{C} \mathrm{NMR}\left(\mathrm{CDCl}_{3}, \mathrm{TMS}\right): \delta$ $163.71(\mathrm{t}, J=27.7 \mathrm{~Hz}), 162.56(\mathrm{t}, J=246.1 \mathrm{~Hz}), 132.49(\mathrm{~d}, J$ $=2.9 \mathrm{~Hz}), 129.94(\mathrm{~d}, J=8.6 \mathrm{~Hz}), 117.81(\mathrm{t}, J=253.7 \mathrm{~Hz})$, 115.88 (d, $J=21.9 \mathrm{~Hz}), 43.15(\mathrm{~s}), 37.84(\mathrm{t}, J=23.8 \mathrm{~Hz}$ ), $29.79(\mathrm{~s}),-2.39(\mathrm{~s}) .{ }^{19} \mathrm{~F} \mathrm{NMR}\left(\mathrm{CDCl}_{3}, \mathrm{CFCl}_{3}\right): \delta-105.77(\mathrm{~s}$, $2 \mathrm{~F}),-119.13$ (s, 1F). IR (KBr); 2959, 1682, 1606, 1512, $1439,1251,1192,1126 \mathrm{~cm}^{-1}$. GC-MS $(\mathrm{m} / \mathrm{z}$, relative intensity): $301(\mathrm{M}+, 3.37), 109(100.0), 83(8.49), 73$ (13.35), 53 (8.79). HRMS: C14H17NOF4Si, Calculated, 301.11098; Observed, 301.11006.

1-Butyl-3,3-difluoro-2-pyrrolidone (7b). $0.82 \mathrm{~g}$ of 1 butyl-3,3-difluoro-5-trimethylsilyl-2-pyrrolidone (3.26 $\mathrm{mmol}), 15 \mathrm{~mL}$ of DMSO and $0.76 \mathrm{~g}$ of $\mathrm{KF}(13.1 \mathrm{mmol})$ were placed in a $30 \mathrm{~mL}$ flask and allowed to react at $100^{\circ} \mathrm{C}$ for $24 \mathrm{hrs}$, or to react along with $6.52 \mathrm{~mL} / 1.0 \mathrm{M}$ THF $(6.52$ $\mathrm{mmol}$ ) of BuNF (tetrabutylammonium fluoride) for $12 \mathrm{hrs}$. The reaction products were extracted by ether, dried over anhydrous $\mathrm{MgSO}_{4}$. Thin layer chromatography (alumina $60 \mathrm{~F}-254$, ethyl acetate: $n$-hexane $=1: 3, \mathrm{R}_{f}=0.67$ ) resulted in separation of $0.41 \mathrm{~g}(2.28 \mathrm{mmol}$ ) of $7 \mathrm{a}$ (yield of $70 \%)$. To prepare $7 \mathrm{c}$ to $7 \mathrm{~h}$, the same procedure was applied, and the yields of products are noted in Table 2 .

'H NMR $\left(\mathrm{CDCl}_{3}\right) \delta: 0.19(\mathrm{t}, 7.3 \mathrm{~Hz}, 9 \mathrm{H}), 1.61(\mathrm{dt}, 7.3 \mathrm{~Hz}$, $2 \mathrm{H}$ ), 1.53 (t, $7.3 \mathrm{~Hz}, 2 \mathrm{H}$ ), 2.48 (dddd, $J=6.7,6.7,15.0,15.0$ $\mathrm{Hz}, 2 \mathrm{H}) .{ }^{13} \mathrm{C} \mathrm{NMR}\left(\mathrm{CDCl}_{3}\right) \delta .13 .8,20.0,28.9,29.4(\mathrm{t}, 22.6$ $\mathrm{Hz}), 41.3$ (br), 43.5, 118.3 (t, $249.5 \mathrm{~Hz}), 163.6$ (t, $30.7 \mathrm{~Hz})$. I"F NMR ( $\left(\mathrm{CDCl}_{3}\right) \& \delta-105.8(\mathrm{dd}, 15.0,15.0 \mathrm{~Hz})$. FT-IR $\left(\mathrm{CCl}_{4}\right): 1262(\mathrm{~s}), 1427(\mathrm{~s}), 1719(\mathrm{~s}), 2973(\mathrm{~s}) \mathrm{cm}^{-1}$. GC-MS $\mathrm{m} / \mathrm{z}$ (rel. intensity): 57 (20.20), 106 (43.83), 120 (14.61), 177 (M+, 37.20). HRMS: C8H13NOF2, Calculated, 177.09652; Observed, 177.09451.

1-Cyclohexyl-3, 3-difluoro-2-pyrrolidone (7e). 'H NMR $\left(\mathrm{CDCl}_{3}\right) \& .1 .11(1 \mathrm{H}, \mathrm{m}), 1.69(1 \mathrm{H}, \mathrm{m}), 1.39(2 \mathrm{H}, \mathrm{m}), 1.75$ $(2 \mathrm{H}, \mathrm{m}), 1.82(2 \mathrm{H}, \mathrm{m}), 2.46(2 \mathrm{H}$, dddd, $6.9,13.3,15.2,15.2$ $\mathrm{Hz}), 3.38(2 \mathrm{H}), 3.97(1 \mathrm{H}, \mathrm{m}) .{ }^{13} \mathrm{C} \mathrm{NMR}\left(\mathrm{CDCl}_{3}\right)$ 8. 25.16 $(2 \mathrm{c}), 25.21(\mathrm{lc}), 29.3(1 \mathrm{c}, \mathrm{t}, 23.0 \mathrm{~Hz}), 29.7(2 \mathrm{c}), 37.2(\mathrm{br}, 1 \mathrm{c})$, $51.7(1 \mathrm{c}), 118.5(1 \mathrm{c}, \mathrm{t}, 249.5 \mathrm{~Hz}), 62.9(1 \mathrm{c}, 31.4 \mathrm{~Hz}) .{ }^{19} \mathrm{~F}$ NMR ( $\left.\mathrm{CDCl}_{3}\right) \delta:-105.99$ (dd, 16.4). FT-IR $\left(\mathrm{CCl}_{4}\right): 1216$ (m), $1431(\mathrm{~m}), 1715(\mathrm{~s}) \mathrm{cm}^{-1}$. GC-MS m/z (rel. intensity): 82 (11.58), $120(0.49), 122(100), 203\left(\mathrm{M}^{+}, 6.12\right)$. HRMS: C10H15NOF2, Calculated, 203.11217; Observed, 203.11228.

1-Benzyl-3,3-difluoro-2-pyrrolidone (7d). 'H NMR $\left(\mathrm{CDCl}_{3}\right) 8.2 .47(2 \mathrm{H}$, dddd, $6.7,6.7,13.3,15.1), 3.30(2 \mathrm{H}$, dd, $6.7,6.7 \mathrm{~Hz}), 4.52(\mathrm{~s}, 2 \mathrm{H}), 7.25(2 \mathrm{H}, \mathrm{m}), 7.35(3 \mathrm{H}, \mathrm{m})$. ${ }^{13} \mathrm{C} \mathrm{NMR}\left(\mathrm{CDCl}_{3}\right) \delta: 29.3(\mathrm{t}, 23.0 \mathrm{~Hz}), 40.8,47.7,118.3(\mathrm{t}$, $250.5 \mathrm{~Hz}), 128.47(1 \mathrm{c}), 128.51(2 \mathrm{c}), 129.2(2 \mathrm{c}), 134.7,163.7$ $(\mathrm{t}, 31.7) \cdot{ }^{19} \mathrm{~F} \mathrm{NMR}\left(\mathrm{CDCl}_{3}\right) \delta .-105.7(\mathrm{dd}, 14.3,14.3)$. FT-IR $\left(\mathrm{CCl}_{4}\right): 1435(\mathrm{~s}), 1262(\mathrm{~s}), 1723(\mathrm{~m}), 2979(\mathrm{~s}) \mathrm{cm}^{-1}$. GC-MS $\mathrm{m} / \mathrm{z}$ relative intensity): $65(15.70), 91(100), 120(0.52), 76$ $(0.73), 211\left(\mathrm{M}^{+}, 34.56\right)$.

$\mathrm{N}$-(2-Phenyl ethyl)-3,3-difluoro-2-pyrrolidone (7e). ${ }^{1} \mathrm{H}$ NMR ( $\left.\mathrm{CDCl}_{3}, \mathrm{TMS}\right): \delta 7.19-7.32(\mathrm{~m}, 5 \mathrm{H}), 3.60(\mathrm{t}, J=7.3$ $\mathrm{Hz}, 2 \mathrm{H}), 3.18$ (t, $J=6.4 \mathrm{~Hz}, 2 \mathrm{H}), 2.91(\mathrm{t}, J=7.3 \mathrm{~Hz}, 2 \mathrm{H}$ ), $2.35-2.41(\mathrm{~m}, 2 \mathrm{H}) \cdot{ }^{13} \mathrm{C} \mathrm{NMR}\left(\mathrm{CDCl}_{3}, \mathrm{TMS}\right): \delta 163.60(\mathrm{t}, J=$ $37.4 \mathrm{~Hz}$ ), 137.96 (s), 128.85 (s), 128.69 (s), 126.95 (s), $117.92(\mathrm{t}, J=243.5 \mathrm{~Hz}), 45.35(\mathrm{~s}), 42.14(\mathrm{~s}), 33.37(\mathrm{~s}), 29.39$ $(\mathrm{t}, J=22.9 \mathrm{~Hz}) \cdot{ }^{19} \mathrm{~F} \mathrm{NMR}\left(\mathrm{CDCl}_{3}, \mathrm{CFCl}_{3}\right): \delta-105.46 \mathrm{IR}$ (KBr); 2991, 1736, 1678, 1614, 1537, 1323, $1273 \mathrm{~cm}^{-1}$. GCMS (m/z, relative intensity): $225\left(\mathrm{M}^{+}, 3.50\right), 134(11.65)$, 104 (90.65), 91 (21.67), 77 (18.12), $42(100.0)$.

$\mathrm{N}$-(3,4-Difluorobenzyl)-3,3-difluoro-2-pyrrolidone (7f). 'H NMR (CDCl 3, TMS): $\delta 6.98-7.18(\mathrm{~m}, 4 \mathrm{H}), 4.47(\mathrm{~s}, 2 \mathrm{H})$, $3.32(\mathrm{t}, J=6.4 \mathrm{~Hz}, 1 \mathrm{H}), 2.46-2.52(\mathrm{~m}, 2 \mathrm{H}) .{ }^{13} \mathrm{C} \mathrm{NMR}$ $\left(\mathrm{CDCl}_{3}, \mathrm{TMS}\right): \delta 163.63(\mathrm{t}, J=31.5 \mathrm{~Hz}), 151.45(\mathrm{dd}, J=$ $249.0 \mathrm{~Hz}, J=13.4 \mathrm{~Hz}$ ), 149.45 (dd, $J=248.0 \mathrm{~Hz}, J=13.4$ $\mathrm{Hz}), 131.71(\mathrm{t}, J=4.7 \mathrm{~Hz}), 124.49(\mathrm{dd}, J=6.7 \mathrm{~Hz}, J=3.8$ $\mathrm{Hz}), 118.04$ (s), 117.90 (t, $J=254.5 \mathrm{~Hz}), 117.34$ (t, $J=17.2$ $\mathrm{Hz}), 46.66(\mathrm{~s}), 40.77(\mathrm{~s}), 29.18(\mathrm{t}, J=24.1 \mathrm{~Hz}) .{ }^{19} \mathrm{~F}$ NMR $\left(\mathrm{CDCl}_{3}, \mathrm{CFCl}_{3}\right): \delta-105.85(\mathrm{~s}, 2 \mathrm{~F}),-136.69(\mathrm{~s}, 1 \mathrm{~F}),-138.88$ (s, 1F). IR (KBr); 2916, 1724, 1620, 1520, 1435, 1284, 
$1261,1116,1010 \mathrm{~cm}^{-1}$. GC-MS (m/ $\mathrm{z}$, relative intensity): 247 $\left(\mathrm{M}^{+}, 24.87\right), 168(6.54), 127(100.0), 101(11.25), 77$ (11.79), $56(6.71), 42(12.29)$.

$\mathrm{N}$-(2-Fluorobenzyl)-3,3-difluoro-2-pyrrolidone (7g). ' $\mathrm{H}$ NMR ( $\left.\mathrm{CDCl}_{3}, \mathrm{TMS}\right): \delta 2.41-2.51(\mathrm{~m}, 2 \mathrm{H}), 3.35(\mathrm{t}, J=6.4$ $\mathrm{Hz}, 1 \mathrm{H}), 4.58(\mathrm{~s}, 2 \mathrm{H}), 7.05-7.36(\mathrm{~m}, 4 \mathrm{H}) .{ }^{13} \mathrm{C} \mathrm{NMR}\left(\mathrm{CDCl}_{3}\right.$, TMS): $\delta 29.19(\mathrm{t}, J=23.8 \mathrm{~Hz}), 40.97(\mathrm{~s}), 47.56(\mathrm{~s}), 115.71$ $(\mathrm{d}, J=21.0 \mathrm{~Hz}), 118.03(\mathrm{t}, J=247.9 \mathrm{~Hz}), 128.36(\mathrm{~d}, J=4.8$ $\mathrm{Hz}), 129.10(\mathrm{~s}), 130.37$ (d, $J=8.5 \mathrm{~Hz}), 130.94$ (d, $J=2.9$ $\mathrm{Hz}), 161.13(\mathrm{~d}, J=245.1 \mathrm{~Hz}), 163.57(\mathrm{t}, J=30.5 \mathrm{~Hz}) .{ }^{19} \mathrm{~F}$ $\mathrm{NMR}\left(\mathrm{CDCl}_{3}, \mathrm{CFCl}_{3}\right): \delta-105.77(\mathrm{~s}, 2 \mathrm{~F}),-119.13(\mathrm{~s}, 1 \mathrm{~F})$. IR (KBr); $1099,1126,1255,1349,1494,1728,2928 \mathrm{~cm}^{-1}$. GCMS (m/z, relative intensity): $56(4.78), 77(3.31), 83(16.69)$, $109(100.0), 150(3.43), 158(2.82), 229\left(\mathrm{M}^{+}, 20.07\right)$.

$\mathrm{N}$-(4-Fluorobenzyl)-3,3-difluoro-2-pyrrolidone (7h). ' $\mathrm{H}$ NMR (CDCl, TMS): $\delta 2.44-2.49(\mathrm{~m}, 2 \mathrm{H}), 3.29(\mathrm{t}, J=6.4$ $\mathrm{Hz}, 2 \mathrm{H}), 4.49(\mathrm{~s}, 2 \mathrm{H}), 7.01-7.06(\mathrm{~m}, 2 \mathrm{H}), 7.20-7.26(\mathrm{~m}, 2 \mathrm{H})$. $\left.{ }^{13} \mathrm{C} \mathrm{NMR}_{(\mathrm{CDCl}}, \mathrm{TMS}\right): \delta 29.17(\mathrm{t}, J=23.8 \mathrm{~Hz}), 40.63(\mathrm{~s})$, $46.88(\mathrm{~s}), 116.08(\mathrm{~d}, J=21.9 \mathrm{~Hz}), 118.06(\mathrm{t}, J=254.0 \mathrm{~Hz})$, $130.17(\mathrm{~d}, J=8.5 \mathrm{~Hz}), 130.49(\mathrm{~d}, J=1.0 \mathrm{~Hz}), 162.71(\mathrm{t}, J=$ $246.1 \mathrm{~Hz}), 163.69(\mathrm{t}, J=32.4 \mathrm{~Hz}) .{ }^{19} \mathrm{~F} \mathrm{NMR}\left(\mathrm{CDCl}_{3}\right.$, $\left.\mathrm{CFCl}_{3}\right): \delta-105.88(\mathrm{~s}, 2 \mathrm{~F}),-114.08(\mathrm{~s}, 1 \mathrm{~F})$. IR (KBr); 1116, $1226,1435,1512,1606,1711,2937 \mathrm{~cm}^{-1}$. GC-MS $(\mathrm{m} / \mathrm{z}$, relative intensity): $57(5.35), 77(3.84), 83(16.25), 109$ $(100.0), 150(5.82), 158(6.31), 229(\mathrm{M}, 23.82)$.

Acknowledgements. This research was funded by Wonkwang University (2005).

\section{References and Notes}

1. Filler, R; Kobayashi, Y. Biochemical Aspects of Fhorine Chenistry; Elsevier Biochemical Press and Kodansha Ltd.: 1982; b) Filler, R. Biochemistry Inolving Carbon-Fhrorine Bonds; ACS: Washington D. C., 1976.
2. Welch, J. J. Tetrahedron $1987,43,3123$.

3. Gelb. M. H.; Svaren, J. P.; Abeles. R. H. Biochem. 1985, 24 , 1814.

4. Burkholder, C. R.; Dolbier, W. R.; Medebielle, M. J. Fhtorine Chent. 2000. 102,369.

5. Davis, C. R.; Burlon, D. J.; Yang Z. Y. J. Fluorine Chem, 1995 $70,135$.

6. Elsheimer, S.; Dolbier, W. R.; Muria, M. J. Org. Chem. 1984, 49, 205.

7. (a) Rong, G.; Keese, R. Tetrahedron Lett. 1990, 31, 5615. (b) Huang. W. Y.; Wang. W.; Hung, B. N. Acta Chimical Sinta (Engl. Ed.) 1986, 178.

8. Hung, W. Y,; Zhang, H. J. J. Fhorine Chem. 1990, 50, 133.

9. Lang, R. W.; Schaud, B. Tetrahedron Lett. 1988, 29, 2943.

10. Watanabe, \$.; Fajila, T.; Sakamoto, M.; Takeda, H.; Kilazume, T.; Yamazaki, T. J. Fltorine Chem. 1997, 82, 1.

I1. (a) Qiu, Z.; Burton, D. J. J. Org. Chent. 1995, 60, 3465. (b) Yang, Z. Y,; Burton, D. J. J. Org. Chem. 1991, 56, 170 . (c) Yang, Z. Y,; Burion, D. J. J. Fluorine Chem, 1989, 45,435.

12. Chen, J.; Hu, C. M. J. Chem. Soc. Perkin Trans. I 1994, 1111.

13. Qiu, Z. M.; Burton, D. J. Tetrahedron Letf. 1994, 35, 1813.

14. (a) Yang, Z. Y.; Burton, D. J. J. Org. Chem. 1992, 57,5144 . (b) Qiu, Z. M.; Burton, D. J. Tetrahedron Left. 1993, 34, 3239.

15. (a) Bystrenina, V. I.; Shebaldova, A. D.; Lizak, I. V.; Sedavkina, V. A.; Krashennikova, M. K. Khim-Farm Zh, 1982, 16(1), 65. (b) Khananashvili, L. M.; Akbobaze, D. Sh.; Dzhaniashvili, L. K.; Lomladize, Z. Sh. Khim-Farm, Zh, 1982, 16(5), 560. (c) Fujii, Y.; Iwasa, H.; Hirai, J.; Takahama, Y; Hasumi, K.; Eriguchi, M. Bioned \& Phamacother 2000, 54, 85. (d) De Meglio, P. G.; Corra, F.; Ravenna, F.; Gentili, P.; Tempra-Gabbiati, G.; Cristina, T.; Riva, M. Famaco. Ed. Sci. 1987, 42(5), 359.

16. Brahms, D. L. S.; Dailey, W. Chem. Rev, 1996, 96, 1585.

17. Okano, T.; Takakura, N.; Nakano, Y.; Okajima, A.; Eguchi, \$. Tetrahedron Lett. 1995. 51.1903.

18. Buck, R. T.; Coe, D. M.; Drysdale, M. J.; Ferris, L.; Haigh, D.; Moody, C. J.; Pearson, N. D.; Sanghera, B. Tetrahedron: Asymmetry 2003, 14, 791.

19. (a) Yang, Z. Y.; Burton, D. J. J. Chem. Soc., Chem. Commun. 1992, 233. (b) Yang, Z. Y,; Burlon, D. J. J. Org. Chem. 1991, 56, 5125 .

20. (a) Kuroboshi, M.; Ishihara, T. Tetrahedron Letf. 1987, 28, 6481. (b) Kuroboshi, M.; Ishihara, T. Bull. Chem. Soc. Jpn. 1990, 63, 428 . 